\title{
Aspectos epidemiológicos e operacionais da vigilância e controle do tracoma em escola no Município de São Paulo, Brasil
}

\author{
Epidemiology and Operational Aspects of Trachoma Surveillance and \\ Control in a School in the Municipality of São Paulo, Brazil
}

\author{
Nilton Harunori Chinen \\ Centro de Prevenção e Controle de Doenças, Coordenadoria \\ de Vigilância em Saúde, Secretaria Municipal de Saúde, \\ Prefeitura da Cidade de São Paulo-SP \\ Suzana A. Funari A. Penteado \\ Núcleo de Epidemiologia, Administração Regional de Saúde 9, \\ Secretaria Municipal de Saúde, Prefeitura da Cidade de São \\ Paulo-SP \\ Jane De Eston Armond \\ Núcleo de Epidemiologia, Administração Regional de Saúde 9, \\ Secretaria Municipal de Saúde, Prefeitura da Cidade de São \\ Paulo-SP

\section{Cármen M. Cleto Duarte An} \\ Núcleo de Epidemiologia, Administração Regional de Saúde 9, \\ Secretaria Municipal de Saúde, Prefeitura da Cidade de São \\ Paulo-SP
}

\author{
Rosa Kazuye Koda D'Amaral \\ Núcleo de Epidemiologia, Administração Regional de Saúde 9 \\ Secretaria Municipal de Saúde, Prefeitura da Cidade de São \\ Paulo-SP \\ Suzana de Jesus Rosa \\ Núcleo de Epidemiologia, Administração Regional de Saúde 9 , \\ Secretaria Municipal de Saúde, Prefeitura da Cidade de São \\ Paulo-SP \\ Wilma T. Miyake Morimoto \\ Centro para Organização da Atenção à Saúde, Secretaria \\ Municipal de Saúde, Prefeitura da Cidade de São Paulo-SP \\ Norma Helen Medina \\ Serviço de Oftalmologia Sanitária São Paulo, Centro de \\ Vigilância Epidemiológica, Secretaria de Estado da Saúde de \\ São Paulo, São Paulo-SP
}

\section{Resumo}

Tracoma é uma ceratoconjuntivite infecciosa que acomete crianças, principalmente. Este trabalho descreve as atividades de vigilância epidemiológica do tracoma em uma escola municipal de São Paulo, Brasil, no período de 1993 a 1997. Realizouse análise dos dados das fichas de investigação epidemiológica. Examinaram-se 7.751 pessoas, entre alunos e funcionários. Anualmente, realizam-se atividades de busca ativa, ações educativas, notificação dos casos, tratamento e controle. As taxas de detecção foram de 4,1\% em 1993, 4,0\% em 1994, 0,1\% em 1995, 0,9\% em 1996 e 0,2\% em 1997. A taxa de alta (cura) foi de $84,0 \%$. Informações sobre saúde não eram enfocadas, regularmente, pelos professores. A intervenção na escola mediante ações educativas, ano após ano, e o tratamento específico reduziram as taxas de detecção. Os autores sugerem que se reavive a Educação em Saúde como tema para o ensino público, na esperança de que as crianças levem aos domicílios o aprendizado obtido na escola, para a conseqüente promoção de saúde na comunidade.

Palavras-chave: tracoma; vigilância epidemiológica; educação em saúde; escolas.

\section{Summary}

Trachoma is a keratoconjunctivitis affecting mainly children. This study describes epidemiological surveillance of trachoma in a municipal school in São Paulo, Brazil, during 1993-1997. Epidemiological investigation forms were analyzed, and 7,751 students and school employees were examined. Active surveillance or active searches, complemented by educational activities, notification of cases, treatment, and control activities were done each year. The detection rate was $4.1 \%$ in 1993, 4.0\% in 1994, 0.1\% in 1995, 0.9\% in 1996, and $0.2 \%$ in 1997 . The cure rate was $84.0 \%$. It was noted that schoolteachers did not regularly focus on health information, and continuous educational activities at the school and specific treatment every year led to a fall in the trachoma rate. The authors suggest emphasizing Health Education as a subject for public schools, with the hope that children would take home lessons learned, strengthening bealth promotion in the community.

Key words: trachoma; epidemiological surveillance; health education; schools.

Endereço para correspondência:

Prefeitura da Cidade de São Paulo, Secretaria Municipal da Saúde, Coordenadoria de Vigilância em Saúde, Centro de Prevenção e Controle de Doenças, Rua Santa Isabel, 181, $7^{\circ}$ Andar, Vila Buarque, São Paulo-SP. CEP: 01221-010

E-mail:nchinen@prefeitura.sp.gov.br 


\section{Introdução}

A cegueira é um importante problema de Saúde Pública que, muitas vezes, não tem recebido a atenção que merece. A maioria das pessoas atingidas por esse mal vive em países em desenvolvimento e está relacionada a infecções, má nutrição e falta de assistência oftalmológica. ${ }^{1}$

0 tracoma é uma ceratoconjuntivite crônica, isto é, afeta a conjuntiva e a córnea. Seu agente etiológico é a bactéria Chlamydia trachomatis, que atinge, mais frequientemente, populações rurais pobres em áreas quentes e áridas de países em desenvolvimento. Tratase da principal causa infecciosa de cegueira evitável. As infecções, repetidas ao longo dos anos, podem evoluir para a formação de cicatrizes que levam à cegueira. A única fonte de infecção é o homem com infecção ativa, qual seja, a forma inflamatória. Crianças de até dez anos de idade e com infecção ativa são os principais reservatórios do agente etiológico nas populações onde o tracoma é endêmico. A principal forma de transmissão é a direta, mão-olho-mão; outra forma é a indireta, por objetos contaminados ou vetores mecânicos. ${ }^{2}$

\section{O tracoma, ceratoconjuntivite crônica que afeta a conjuntiva e a córnea, é a principal causa infecciosa de cegueira evitável.}

A Organização Mundial da Saúde (OMS) estima que existam 84 milhões de pessoas com tracoma, das quais 1,3 milhões totalmente cegas em razão da cicatriz corneana. ${ }^{3}$ A doença está relacionada a baixas condições socioeconômicas e locais sem saneamento básico. A questão da higiene pessoal é de fundamental importância na prevenção, especialmente na lavagem das mãos e do rosto, para romper a cadeia de transmissão da infecção. Em países desenvolvidos, seu controle foi alcançado, principalmente, pela melhoria das condições sanitárias. ${ }^{4}$

O Estado de São Paulo foi uma das regiões de disseminação do tracoma no Brasil, a partir da imigração européia no século XIX. ${ }^{5}$

No inquérito epidemiológico de âmbito nacional realizado na década de 70 , com o objetivo de conhecer a distribuição geográfica da endemia de tracoma e recomendar as áreas prioritárias para programas de controle, as unidades da federação que apresentaram maior prevalência da doença foram o Pará $(26,2 \%)$, o Amazonas (15,7\%) e Roraima (14,6\%), mostrando que, amplamente disseminada no país, estava erradicada, praticamente, no Estado de São Paulo, onde apresentava prevalência de $0,61 \%$ entre a população escolar. Hoje, o tracoma é encontrado em todo o território nacional; sob vigilância epidemiológica, tem status de doença de notificação obrigatória nos Estados de Mato Grosso, Ceará e São Paulo;, ${ }^{5,6}$ neste, se 0 tracoma voltou a ser doença de notificação compulsória a partir de 1992,,8 a década de 80 já assistia a um ressurgimento de casos da doença, em número crescente, no interior do Estado, embora apenas em 1990, os primeiros casos na cidade de São Paulo foram detectados no bairro de Brazilândia, zona norte da capital.

Dados do Sistema de Vigilância Epidemiológica da Secretaria de Estado da Saúde (SES/SP) mostram taxas de prevalência no Município de São Paulo de 4,6 por 100.000 habitantes em 1993, 16,1/100.000 em 1994, 6,1/100.000 em 1995, 2,3/100.000 em 1996, 3,3/100.000 em 1997 e 3,4/100.000 em 1998. ${ }^{9}$

0 presente trabalho tem como objetivos descrever as atividades de vigilância epidemiológica em uma escola de primeiro grau e analisar os dados das fichas de investigação epidemiológica (FIE) de tracoma no período de 1993 a 1997, para conhecer os fatores que interferiram na taxa de detecção da doença.

\section{Metodologia}

o Programa de Investigação Epidemiológica do Tracoma pela Administração Regional de Saúde 9 (ARS 9), da Secretaria Municipal de Saúde de São Paulo(SMS/SP), teve início em 1993, após notificação de um caso de tracoma confirmado por exame clínico e por teste de imunofluorescência direta para Cblamydia trachomatis.

A escola municipal de primeiro grau em estudo, que pertence ao Distrito Administrativo (DA) de Pedreira, área de abrangência da ARS 9, é abastecida de água e esgoto pela rede pública e está situada na região sul do Município de São Paulo, este constituído de 96 distritos administrativos. 0 DA de Pedreira apresenta índice de exclusão/inclusão social (IEX) de 0,72 , pelo qual ocupa $074^{\circ}$ lugar entre os IEX dos DA. ${ }^{10}$ 
A partir da notificação de um caso de tracoma, foi desencadeada busca ativa entre os alunos, professores e funcionários presentes na escola durante os anos letivos de 1993 a 1997. Os comunicantes domiciliares dos casos positivos foram convocados para exame, ocasião em que se preencheram as fichas de investigação epidemiológica (FIE), iniciou-se o tratamento e a orientação dos casos de tracoma inflamatório. A visita domiciliar foi realizada para exame dos comunicantes faltosos, como também para conhecer as condições de habitação desses casos.

Para a análise, foram utilizados dados das FIE: identificação; manifestação clínica; inflamação tracomatosa; conjuntivite associada; contato com casos semelhantes; comunicantes de casos clínicos; e condições de saneamento do domicílio.

Os profissionais da equipe de saúde foram treinados e padronizados para o diagnóstico de tracoma pelo Centro de Vigilância Epidemiológica (CVE) e pelo Centro para Organização da Atenção à Saúde (COAS). 0 treinamento consistiu de parte teórica, com apresentação de temas relacionados à saúde ocular, anatomia, doenças oculares - como tracoma (definição, diagnóstico, tratamento e controle) - e orientação sobre 0 preenchimento das fichas. A parte prática do curso consistiu de busca ativa em escolas selecionadas previamente; e de realização, pelos treinandos, de exame clínico em crianças normais e positivas para tracoma. Considerou-se padronizado aquele profissional que apresentasse um percentual de acerto no diagnóstico de $80 \%$ dos casos. ${ }^{11}$

A equipe local, uma vez treinada, realizou as ações educativas nos três períodos escolares; utilizou-se de recursos audiovisuais - apresentação de diapositivos (slides) e álbuns seriados sobre a doença-, distribuição de panfletos e demonstração prática da técnica de eversão palpebral no exame clínico. Com o intuito de avaliar 0 aproveitamento das palestras, foram aplicados questionários de perguntas dissertativas e de múltipla escolha. Durante as palestras, os pais ou responsáveis foram solicitados a explicitar, por escrito, na caderneta de anotações dos alunos, quando não autorizassem a submissão da criança ao exame.

Para a busca ativa, definiu-se como caso de tracoma qualquer pessoa que, a exame ocular externo, apresentasse, pelo menos, um dos sinais do Esquema Simplificado de Gradação da OMS, ${ }^{12}$ a saber:
- tracoma inflamatório folicular (TF), quando há, pelo menos, cinco folículos de $0,5 \mathrm{~mm}$ de diâmetro como mínimo;

- tracoma inflamatório intenso (TI), quando mais de $50 \%$ dos vasos tarsais profundos encontram-se obscurecidos por hipertrofia papilar e edema;

- tracoma cicatricial (TS), quando há presença de cicatrizes conjuntivais;

- triquíase tracomatosa (TT), quando há, pelo menos, um cílio tocando o globo ocular ou evidência de cílios epilados; ou

- opacificação corneana (CO), quando a opacificação, facilmente visível, atinge a área pupilar.

0 tratamento preconizado consistiu na aplicação de tetraciclina $1 \%$ pomada oftálmica - fornecida pela SES/SP - duas vezes ao dia, por 45 dias consecutivos, com retorno do paciente após esse período para 0 primeiro controle de tratamento. A cada 45 dias, novos retornos foram realizados para controle de cura, em total de três. Se, durante esse período, não houve intercorrência de tracoma inflamatório (TF-TI), nem cicatrizes conjuntivais (TS), o paciente recebeu alta - curado, sem cicatriz. Quando apresentou cicatrizes, recebeu alta - curado, com cicatriz - e orientação para retornar uma vez ao ano, para controle. ${ }^{13}$

Reforçaram-se as seguintes medidas de prevenção: lavar o rosto sempre que lavar as mãos; separar e manter a higiene de objetos de uso pessoal; utilizar toalhas descartáveis na escola; e tomar os devidos cuidados para diminuir a população de moscas e mosquitos (cuidados com lixo, resto de comida, entre outros).

A taxa de detecção na instituição foi calculada pelo número de casos positivos em relação ao total de examinados multiplicado por cem, no período de $1^{\circ}$ de janeiro a 31 de dezembro de cada ano. ${ }^{6}$

Os autores ressaltam que essa atividade constitui rotina da vigilância epidemiológica, em que é sempre solicitada a autorização dos pais para a realização dos exames dos escolares.

\section{Resultados}

Foram examinadas 7.751 pessoas, entre alunos, professores, funcionários e comunicantes de casos positivos de tracoma no período de 1993 a 1997. Todos os comunicantes do caso-índice foram examinados, não tendo sido encontrado qualquer caso positivo de tracoma. 
A Tabela 1 apresenta os casos notificados, os examinados e a taxa de detecção observada na escola de primeiro grau, segundo presença de inflamação ocular de origem tracomatosa e anos de ocorrência. As taxas de detecção indicam declínio no decorrer dos cinco anos estudados, a partir de intervenção na escola com ações educativas, ano após ano, e instituição do tratamento preconizado. Essas taxas foram, proporcionais, inversamente, ao número de pessoas examinadas durante o período de 1993 a 1997; assim, quanto maior o número de examinados, menor o número de casos clínicos detectados.

Em relação ao tipo de tracoma, 95,4\% (125) dos casos diagnosticados eram do tipo folicular (TF),
$3,8 \%$ (5) do tipo cicatricial (TS) e $0,8 \%$ (1) do tipo intenso (TI).

A distribuição dos casos segundo o sexo foi de $41,2 \%$ (54) para o sexo masculino e de 58,8\% (77) para o feminino.

0 percentual dos casos de tracoma folicular foi menor nos grupos etários extremos, de 1 a 4 anos $(1,6 \%)$ e de 50 anos ou mais $(0,8 \%)$; a maioria desses casos concentrou-se nas faixas etárias de 5 a 9 e de 10 a 14 anos, totalizando mais de $80 \%$ dos casos investigados (Figura 1).

Os casos de tracoma cicatricial (TS) ocorreram de acordo com a seguinte distribuição proporcional por faixa etária: $60,0 \%$ (3) de casos de 20 a 49 anos;

\section{Tabela 1 - Casos de tracoma notificados, número de examinados e taxa de deteç̧ão em escola do Município de São Paulo-SP. Brasil, 1993 a 1997}

\begin{tabular}{lccc}
\hline Ano & Número de casos de tracoma & Número de examinados & Taxa de deteç̧ão (\%) \\
\hline 1993 & 47 & 1.138 & 4,1 \\
1994 & 61 & 1.520 & 4,0 \\
1995 & 2 & 1.456 & 0,1 \\
1996 & 17 & 1.822 & 0,9 \\
1997 & 4 & 1.815 & 0,2 \\
\hline
\end{tabular}

Fonte: Prefeitura da Cidade de São Paulo, Secretaria Municipal de Saúde, Administração Regional de Saúde 9

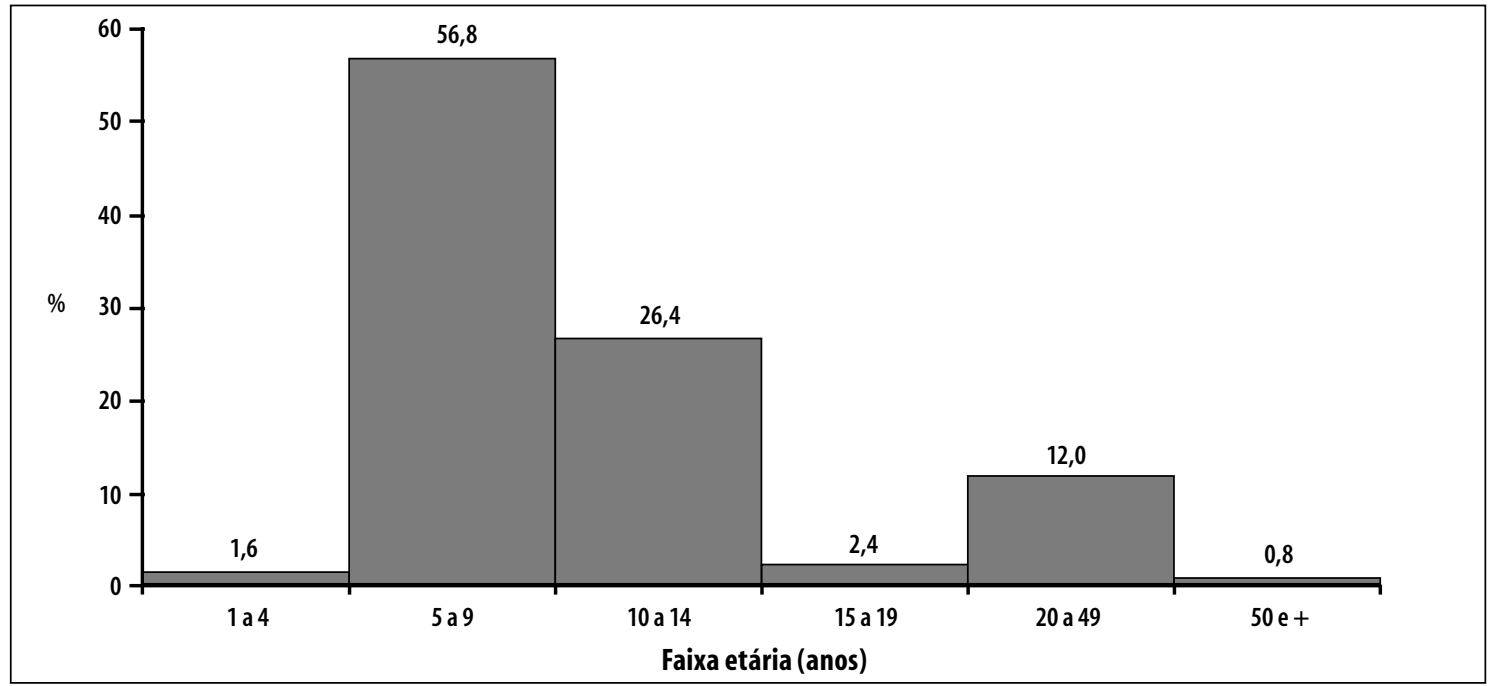

Fonte: Prefeitura da Cidade de São Paulo, Secretaria Municipal de Saúde, Administração Regional de Saúde 9

\section{Figura 1 - Distribuição dos casos notificados de tracoma folicular por faixa etária em escola do Município de São Paulo-SP. Brasil, 1993 a 1997}


20,0\% (1) de 15 a 19 anos; e 20,0\% (1) acima dos 50 anos. 0 único caso de tracoma intenso (TI) foi de uma criança com idade entre 5 e 9 anos.

As manifestações clínicas, presentes em 73,6\% dos casos, foram as seguintes mais freqüentes: prurido $(44,3 \%)$; lacrimejamento $(30,5 \%)$; hiperemia $(28,2 \%)$; ardor $(23,7 \%)$; sensação de corpo estranho (17,6\%); e fotofobia (11,5\%).

Verificou-se que $98,5 \%$ (129) dos casos não apresentaram associações com conjuntivites; e que a taxa de alta - curado - foi de $84,0 \%$ para o período em estudo.

Em relação aos comunicantes, 47,6\% (291) foram examinados e 27 casos de tracoma (9,3\%) foram diagnosticados. Os autores ressaltam que, no ano de 1997, todos os comunicantes foram examinados e nenhum caso foi detectado.

0 tipo de contato predominante com casos semelhantes foi em creche/escola, para 89,3\% (117) dos casos. Somente para 9,2\% (12) dos casos, o contato foi domiciliar; para 1,5\% (2), não havia essa informação.

Quanto ao abastecimento de água, verificou-se que, em 98,5\% (129) dos domicílios onde ocorreram os casos, a água era fornecida pela rede pública - menos de $2 \%$ do abastecimento dependia de outras fontes, portanto. Sobre esgotamento sanitário, 53,9\% (69) dos domicílios encontravam-se conectados à rede pública e 46,1\% (59) não, conforme demonstra a Figura 2.

\section{Discussão}

0 impacto da doença foi brando nessa escola. Detectou-se apenas um caso de TI em 1994 e não foram encontrados casos de TT e CO, além de haver pouca associação com conjuntivite, fatores agravantes do prognóstico.

Observou-se a ausência de predominância significativa em relação ao sexo, em concordância com outros trabalhos nacionais. ${ }^{14-16}$

Os casos de tracoma folicular ocorreram, com maior frequiência, na faixa etária de 5 a 14 anos, dados também coincidentes com os da literatura em que crianças são apontadas como o principal reservatório para a transmissão da doença. ${ }^{16}$ No tocante ao tracoma cicatricial (TS), vale ressaltar que quatro desses casos eram migrantes dos Estados da Bahia, do Ceará, de Minas Gerais e de São Paulo (interior deste Estado); apenas para um caso, não foi possível identificar a

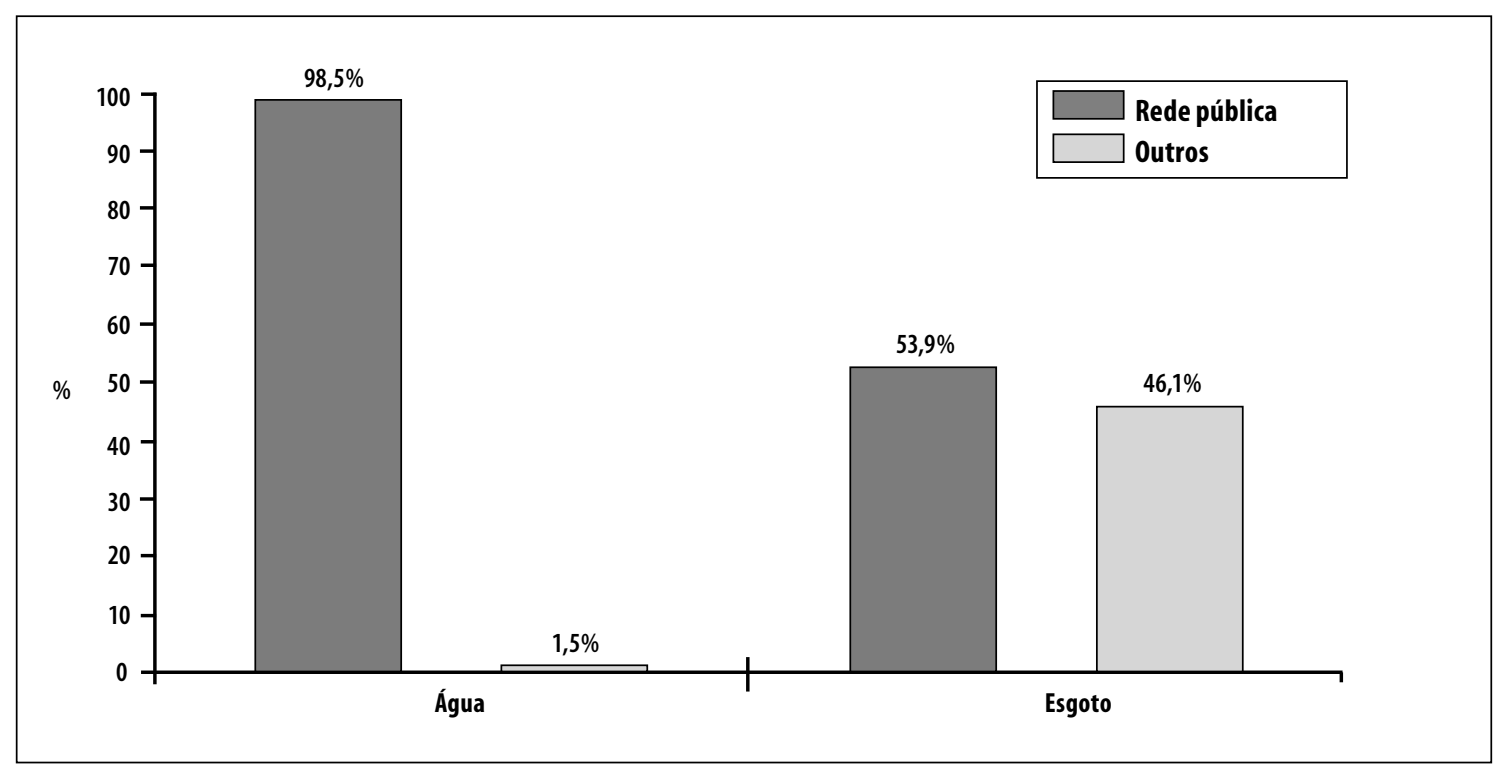

Fonte:Prefeitura da Cidade de São Paulo, Secretaria Municipal de Saúde, Administração Regional de Saúde 9

\section{Figura 2 - Distribuição dos casos notificados de tracoma segundo abastecimento de água e esgoto em escola do Município de São Paulo-SP. Brasil, 1993 a 1997}


procedência. Esses fatos sugerem que o tracoma seja um acontecimento recente na região, haja vista a observância de maior frequiência de tracoma folicular e de casos de TS em indivíduos não originários da área da ARS 9. ${ }^{17}$

Grande parte dos casos apresentou algum tipo de sintoma, o mais freqüente o prurido, resultado semelhante ao obtido em estudo realizado no povoado de Poço Redondo, Estado da Bahia. ${ }^{18}$

\section{A educação em saúde e a intervenção medicamentosa promoveram uma redução quantitativa do agente etiológico circulante, elevada taxa de cura e queda na taxa de deteç̧ão da doença na escola.}

A escola agrega a faixa etária mais acometida pelo tracoma que apresenta, como principal modo de transmissão, o contato direto mão-olho-mão. A ocorrência de casos, a ausência de detecção em alguns domicílios e a referência de contato com casos semelhantes na escola são evidências que indicam a instituição como local facilitador da disseminação da doença. A lavagem das mãos e do rosto com frequiência, fator importante na prevenção, faz com que se quebre a cadeia de transmissão da Chlamydia trachomatis. A escola é abastecida de água - potável e de boa qualidade - pela rede pública, além de dispor de esgoto sanitário. 0 número de torneiras disponíveis para lavar as mãos no período de recreação, entretanto, pode não ser suficiente; se todos os alunos saem para o recreio ao mesmo tempo, seu acesso à água é dificultado. Trabalho desenvolvido no Município de Bebedouro, Estado de São Paulo, demonstrou que existe uma relação entre ocorrência de tracoma e acesso a água; observou-se que, nas casas que contavam com água em seu interior, o número de casos detectados era menor, comparativamente àquelas cuja fonte de água era externa. ${ }^{19}$

As atividades de vigilância epidemiológica do tracoma realizadas, continuamente, nessa escola, em especial as atividades de educação em saúde para a prevenção e o tratamento da doença, e a intervenção medicamentosa, promoveram uma redução quanti- tativa do agente etiológico circulante e, conseqüentemente, elevada taxa de cura e queda na taxa de detecção para menos de 1\%. 0 sincronismo dessas ações permitiu que se controlasse a doença.

Para prevenir o aparecimento de novos casos, os autores sugerem à escola a instalação de mais torneiras no pátio e/ou a programação da recreação dos estudantes em períodos escalonados - algumas classes de cada vez -, além de manter as atividades de educação em saúde.

Os resultados desse trabalho demonstram que a simples oferta de água não deve ser o foco principal das ações dirigidas para o controle da doença; é primordial, igualmente, a inclusão de hábitos de higiene no cotidiano das pessoas, especialmente nas áreas de limitada inclusão social.

No período deste estudo, o tópico Educação em Saúde não constava da grade curricular. Trabalho nesse sentido foi iniciado na década de 70, com o Programa de Oftalmologia Sanitária Escolar para as escolas sob administração do Estado de São Paulo. Reunido em um manual educativo, o Programa, entretanto, não abrangeu as escolas municipais. ${ }^{20}$ No final de 1997 , a Secretaria de Educação Fundamental do Ministério da Educação e Cultura lançou o Programa Curricular Nacional (PCN), para subsidiar as escolas estaduais e municipais na elaboração de conteúdo curricular. ${ }^{21} 0$ assunto foi contemplado em temas transversais, entre eles a Saúde, o que não significou a criação de novas áreas ou disciplinas e sim temas que permeariam as disciplinas convencionais, todavia não obrigatórios.

É fundamental, portanto, a conscientização dos professores sobre a importância da educação em saúde nas salas de aula, cujo desenvolvimento dar-se-ia em duas etapas: a primeira, fixa, abordaria noções básicas de higiene e manutenção da saúde; e a segunda, variável, enfocaria doenças que afetassem a região em determinado momento - por exemplo, tracoma e/ou sarampo e/ou leptospirose e/ou hepatite e/ou dengue, entre outras. A efetivação desse processo dependeria, outrossim, da criação de um sistema de parceria entre as áreas da Saúde e da Educação que dinamizasse o impacto dos conhecimentos transmitidos às crianças para prevenção da ocorrência de doenças; elas, na idéia proposta por estes autores, levariam o conhecimento aprendido para dentro dos seus lares, ampliando a difusão de novos conceitos e hábitos para a promoção de saúde na sua família e comunidade. 


\section{Referências bibliográficas}

1. Organización Mundial de la Salud. Estrategias para la prevención de la ceguera en los programas nacionales: un enfoque desde el punto de vista de la atención primaria de salud. $2^{\text {a }}$ ed. Ginebra: OMS; 1997.

2. Dawson CR, Jones BR, Tarizzo ML. Guide to trachoma control in programmes for the prevention of blindness. Geneve: World Health Organization; 1981.

3. Resnikoff S, Pascolini D, Etya'ale D, Kocur I, Pararajasegaram R, Pokharel G, Mariotti S. Global data on visual impairment the year 2002. Bulletin of the World Health Organization 2004;8:811-890.

4. Taylor HR, Velasco FM, Sommer A. The Ecology of trachoma: an epidemiological study, in Southern Mexico. Bulletin of the World Health Organization 1985;63:559-567.

5. Freitas CA. Prevalência de tracoma no Brasil. Revista Brasileira de Malariologia e Doenças Tropicais 1976;28:227-380.

6. Fundação Nacional de Saúde. Manual de controle do tracoma. Brasília: Ministério da Saúde; 2001.

7. Campos CEG. Prevalência de tracoma entre préescolares e escolares, moradores em favelas do bairro da Freguesia do Ó, região norte do Município de São Paulo - Estado de São Paulo [tese de Mestrado]. São Paulo (SP): Escola Paulista de Medicina; 1992.

8. Secretaria de Estado da Saúde de São Paulo. Resolução S.S. 60/92 de 17/02/92, alterada. Diário Oficial do Estado 104(162), p.21, 1994.

9. Secretaria de Estado da Saúde de São Paulo. Centro de Vigilância Epidemiológica. Dados do Sistema de Vigilância Epidemiológica do Tracoma. São Paulo: Secretaria de Estado da Saúde; 1998.

10. Sposati A. Mapa da exclusão/inclusão social da cidade de São Paulo/2000. Dinâmica social dos anos 90 [monografia em CD-ROM].

11. Organização Mundial da Saúde. Programa para a prevenção da cegueira. Procedimentos para o controle do Tracoma em nível de atenção primária da saúde. OMS/PBL/93.33. Genebra: OMS; 1993.

12. Thylefors B, et al. A Simple system for the assessment of trachoma and its complications. Bulletin of the World Health Organization 1987;65:477-483.
13. Secretaria de Estado da Saúde de São Paulo. Centro de Vigilância Epidemiológica. Manual de vigilância epidemiológica do tracoma: normas e instruções. São Paulo: Centro de Vigilância Epidemiológica; 1993.

14. Luna EJA, Medina NH, Oliveira MB. Vigilância epidemiológica do tracoma no Estado de São Paulo. Arquivos Brasileiros de Oftalmologia 1987;50(2):7079.

15. Medina NH, Gentil RM, Oliveira MB, Sartori MF, Cabral JH, Vasconcelos MS, Barros OM. Investigação epidemiológica do tracoma em pré-escolares e escolares nos Municípios de Franco da Rocha e Francisco Morato. Arquivos Brasileiros de Oftalmologia 1994;57(3):154-158.

16. Nóbrega MJ, Bonono PPO, Scarpi MJ, Guidugli T, Campos CEG, Juliano Y, Novo NF. Prevalência de tracoma em crianças pré-escolares e escolares da periferia da cidade de Joinville, Estado de Santa Catarina, Brasil. Arquivos Brasileiros de Oftalmologia 1993;56:13-17.

17. Medina NH, Massaini MG, Azevedo CLB, Harima C, Prado M, Maluf S, Marcucci M, Caligaris LSA, Morimoto WTM. Vigilância epidemiológica do tracoma em instituição de ensino na cidade de São Paulo, SP. Revista de Saúde Pública 1998;32(1):5963.

18. Scarpi MJ, Gentil R. Sinais e sintomas do tracoma em povoado do Estado da Bahia - Brasil. Arquivos Brasileiros de Oftalmologia 1990;53(6): 276-278.

19. Luna EJA, Medina NH, Oliveira MB, Barros OM, Vranjck A, Melles HHB, West S, Taylor HR. Epidemiology of trachoma in Bebedouro, State of São Paulo, Brazil: prevalence and risk factors. International Journal of Epidemiology 1992;21:169177.

20. Secretaria de Estado dos Negócios da Educação de São Paulo. Oftalmologia sanitária escolar - aspectos educativos. São Paulo: Secretaria de Estado dos Negócios da Educação; 1974.

21. Ministério da Educação. Secretaria de Educação Fundamental. Parâmetros curriculares nacionais: apresentação dos temas transversais. Brasília: MEC/ SEF; 1997. 